Panfoum buIbostun ${ }_{11}$

787

Stipa

523

1345

355

431

806

522

502

926

Blepharoneurou tricholepis (Torr.) Nesh

832 Juhlenbercia rigons (Bentli.) Hitche.

640 Arrostis atolonlfere L;

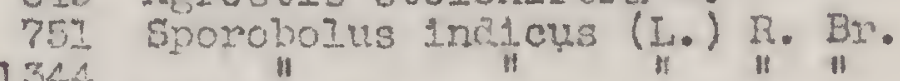

1344

1249

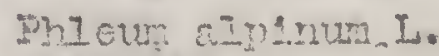

521 Artstida

1059

1.076

1251

1354

607

973

648

1172

674

1171

1088

1169

703

543

527

913

873

879

$\sqrt{594}$

i1 $\operatorname{arczonica}$ Vascy ?

Andiopocon secoharoudes sit.

Pespalun publforum Runr.

Diglterla sengulnelis (I.) Scop.

Br1za rotuncate (His.) Stend.

Penicum nocatur H. \& C.

" obtusur IEK.

Elymirs Anterruptus Bucls.

Sitarion publfioxum J. G. Snith

Argropyron arizonicum scrib. \& Smith

Descherpsta

808

932

811

812

545

1078

1168

1058

667

1345

679

366

1116

362

1248

448

Trisetum

Koeleroia cristata (L.) Pers.

Bromus

$\underset{\text { purgens }}{\text { L. }}$

Iacinletus Beal

Eragrost1s

$$
\begin{aligned}
& \text { "I } \\
& \text { Iugena Nees }
\end{aligned}
$$

Brizà rotundata. (HBK.) Steud.

Poa annua $L$.

" nervose (Hool.) Vasey 
$80 ?$

809

810

1131

544

591

699

692

J 480 495

1194

777

621

835

295

726

1256

359

300

470

458

296

280

288

513

724

504

814

835

557

419

344

1250

663

531

352

4.5

854

548

1262

795

467

722

821

839

889

734

543

670

937

678

1258

669

441

890

761

556

Poa

Festuca ovina. L.

Priva mezicana. (L.) Pers.

*

Samuela carnerosana Trelease

Agave varlefata Jacobl

Tradescant1a

$$
\begin{aligned}
& \text { Karvingtyana R. \& }{ }_{\|} \text {" } \\
& \text { Pringle1 "ateon }
\end{aligned}
$$

Anthericum stenocarpum Bar.

"Nothoscordun bivalve (L.) Eritton

zieanenus v1rescens (MRK.) Macbr.

Zephy inthes Lindleyants Homb.

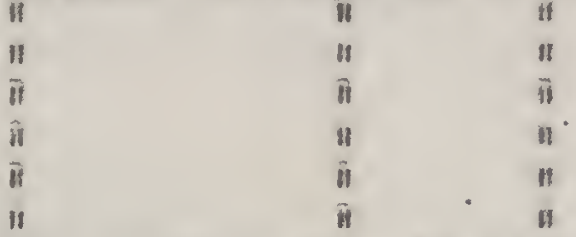

Sabedilia officinalig (\$. \& c.) Standl.

siayrinchim scabmin ch. \& schd?.

" " " " " " "

Vemonia Irvendberepil Gray

Chrysopsis v117.03a Nutt.

Actinea lusimis (Gray) Ktze.

Bldens pliosa L.

zexmenla hf. aptede (HBK.) Grey

det. B. B. Drew

Ps11actis teruis watson

Ertereron

Cirsium fild ondulun Engelm.

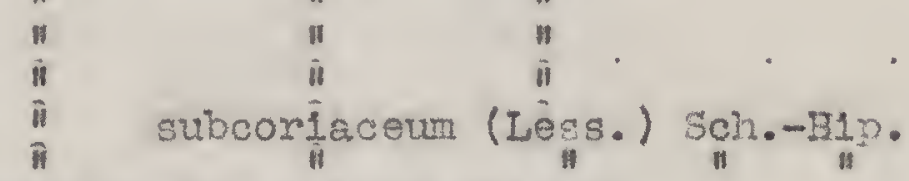

Pyrihopenous multicaulis DC.

Tarezecum mex1canum DC.

Tothonla tubeeformis Cass.

Zexmenia hispida (HBK.) Gray

det. $\underset{n}{\text { Blake }}$

Helenfum oocinfun Gray

" integrifolium (HBK.) B. \& H.

Sclerogemus unserial1s (Hook.) ${ }_{\|}$. \& H.

Gnaphaliun

oxyphyl Iura DC.

purpureur L.

det. Blake 
682

990

409

533

953

910

278

. 1253

337

353

705

782

896

764

795

1155

574

268

859

1164

635

1324

271

1014

$+783$

769

001

1154

696

294

403

285

331

$100 \%$

816

371

1020

51.5

648

1026

1140

348

416

383

357

1247

774

1255

368

690

900

1196

1267

789

484

569

430

1110

720

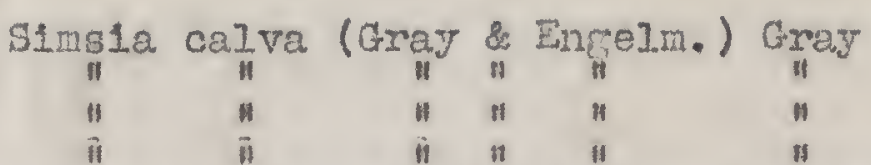

Verbes1na Rothrocic1 Rob1nson \& Greenman hypomelaca Robınson \& Greenman

Psilactia tenuls Watgon

Bidens

Actinee linearlfoila (Hook.) Ktze.

Hellanthella n. sp. ?

! Biake

Heterotheca Lamarcki Cass.

Grindelia oxylepls Greeno

Phorotrichis BeIbisil Grar

Teucring cubeng $\mathrm{se}$

Stachjz nevetếrolla Deș.

Iedeome quinguenervata Bartiat

Ilonarda citriodora Cerv.

Prunelia vicaris i.

Soutejlarie Drum ond 1 Benth.

Arlstolochla brevipes Benth.

Iyomo ea

Arolemias birentata Jomel.

-1aucescens MBK.

tiberone. Ia.

iो brachystephens Enmelm.

"I

in

"

Funa.t.um crianum Sohltr.

Asclepsociora

iI

ค่

Letsstelma engustifollum Turç.

Pherotrichis Balb1s:1 Cray

Gonolobus ret1culatus Enrelm.

Sencc1o cyclophyllus Groenman

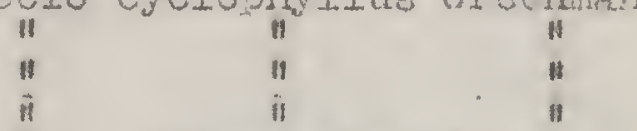

ii vilneraria DC.

"

ii "

"

Evolvulus f111pes ilart.

Iaurand1a Barolayana Lind.

Convolvulus incanus Vahl.

D1chondra argentea $\nabla 111$.

$$
\text { " repens Forgt. }
$$

Cuscute. 
901

913

841

921

1027

1205

779

870

334

860

345

790

1246

570

1137

778

404

855

602

432

346

328

559

440

1134

1132

794

838

981

1207

660

$3 \leq 7$

342

341

867

498

619

360

1015

1081

804

775

738

1233

597

899

299

1067

907

686

865

298

1252

982

291

279

525

505

Tausch1a

$$
\text { " }
$$

lusenfopsis ternatus (Watgon)

Bouchetla anomala Britton \& Rusby

$\begin{array}{lllll}\text { "i } & \text { " } & \text { " } & \text { " } \\ \text { i" } & \text { i } & \text { " } & \text { " } & \text { " } \\ \text { i } & \text { i" } & \text { i } & \text { " }\end{array}$

G111à plnnata (Cav.) Brand

Polemonlum pauciplorum. Watson

Loesella scarlose (Mart. \& Gal.) MaIp.

Physal1s 1rocarpa Brot.

Solanun dejectum Fernala

$$
\text { " " " " }
$$

Micòtlane nuàlcaulis Watson

Physalis

Verbena clliata Benth.

$$
\text { " canescens HBR. }
$$

Eryngium Deppeanun Ch. \& Schdi.
iิ
sp.
" serratur Cav.

Sanicula mexlcana DC.

Coriandrun sat1vum L.

Oenothera rosea M1t. det. Munz speciosa Iutt. approaching var, Berlandieri (Spach)Munz lac1niata var. pubescens (W111a.) llunz tetraptera Cav. Kunth1ana (Spach) Iunz

Gaura

Denothera macroscoles Gray.

"Iuelierl ined. Munz 1935.

Coryầalis aurea W111a.

Geranlum crenatum Watson

Delphinium azureum $111 \mathrm{chx}$. var.

Thal1ctrum

Renunculus geoldes HBK.

Capsella Schaffner1 Natson

Thelypodium

crenthum (Gray) Watson

s1symbrium eur1culatum Gray

Cardamine aurfculate Watson

Draba

Polysala

Lesquerelia

"1"

Erysimum

Dendler1 Watson

det. Rossbach 
640
601

det. Rossbach
"

$4 \leq 9$

318

735

929

262

818

285

Cotyledon

ii Schaffners "atson

Sedum moranense HBK.

"Palmert Watson

1236

Heuchera mexicana Schaffner

611

1220

Phynchosia?

831

823

275

La thyms

Astragalus

564

$565 \AA$

1257

1254

813

717

912

828

571

693

689

498

1312

645

936

460

492

1045

712

1184

1318

905

1192

951

914

1049

714

Lupinus

hypoleucus Schauer

II

Potentilla obovat1folla Ryab. ?

Potentilia

Fragarla mexicane Schal.

Passiflora exsudans Zuce.

V1ola flngelliformis Hemsl.

Hybanthus verbenaceus (HDK.) Loes.

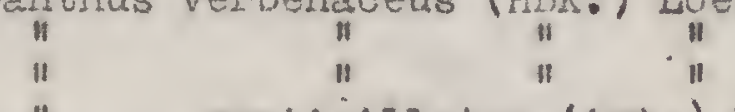

$"$ vertic1llatus (Art.) Ba1lI.

Boehmeria cylincirica (L.) Sw.

Parietaria debilis Forst.

Eriogonu undulatun Benth. atrorubens Engein.

Rumex crispus L.

Froelichia interrupta lloq.

Celosia peniculata L.

Gomphrena decumbens Jacq.

Boerharvia Grac1IIIma Helmerl.

Oxybaphus aggreggatus $\operatorname{Vahl}_{n}$

Phytolacca octandra L.

1111 Portulaca oleracea L.

1129

1108

1117

273

776

1023

1240

946

780

983

507

1019

526

1204

Talinur potens Willa.

Drymar1a

Arenarla decussata Villa.

Stellarla

S1leno laciniata Cav.

$$
\text { Polycala see Blake }
$$


560

566

443

338

469

470

916

1022

906

1029

314

302

852

493

558

964 462

$1085 \mathrm{~A}$

301

270

1320

$104 ?$

641

509

293

664

635

963

405

$+892$

1038

496

827

659

908

349

1046

456

791

272

483

305

402

$3 z 0$

561

577

1061

792

1319

715

591

874

922

651

824

277

417

520

1041

Polygala
"

Linum Schiedeanum Ch. \& Schdl.

$$
\text { " }
$$

"

$$
\text { scabrellum Planch. }
$$

Lev1si1 Pursh

oxal1s latifolia HBK.

$$
\text { Vrighti1 Gray }
$$

$$
\text { " }
$$

Grahaniana Benth.

Geran1um crenatum wats.

Erodium c1cutarium LiHer.

Hyper1oum

Calinrrhoe Iineariloba Gray

Acalypha phleoides Cav.

ii ostryaefolia R1dd.

Phylienthus polyEonoldes spreng.

Euphorb1a

$$
\text { Eraminea Jecq. }
$$

Lossẹlla coerulea (Cav.) G. Don

Ihandevilla Kartinsic11 (Muell.-Arg.) Hemsl.

Cerex.

Commeline gramintólia HBK.

Tradescentia crassifolla Cav.

Anagalis arventis I.

Asclepias long1cornu Benth. galloides 1 "BB.

Conophol1s americana wallx.

Orobanche

Campanula rotumaifolia ".

Lobelia sublibere "ats.

$$
\text { " Bertendier1 DO. }
$$

Specularia perfoliata A. DC.

Tetramerium

Plantago Galeottiane. Dcne.

$$
\text { " Deppeane Vatke }
$$

Valerianella lent

Galiun uncinnulatum DC. ? too young

Houstonia Firightil Gray

Ionglpes Watson rubra Cav. 



\section{thar. 11,1936}

\section{Luelior Plants}

293 Euphorbia trichocardia. B. B. Smith, n. sp.

355 Stipa leucotricha Trin. \& Rupr.

37 Asclep1odora zanthodacryon L. B. Sm1th, n. ap.

437 Stipa mucronata HBK.

443 Polygala alba Nytt. var. tenulfolla (Pursh) Blake

507

" $"$ "

"

n $n$

514 Ascieplodorà zanthodaçryon L. B. Smith,

560 Polyçala albe Nutt. var. tenulfolla (Pursh) Blake

566

"

11

u

n

n

"1

780

iิ

iิ

ii

|ो

n

806 Stipa Pringlel Scribn.

816 Ascleplodora zanthodacryon L. B. Smith

892 Carex liackenziana "eatherby, n. sp.

1019 Polygala alba Nutt. var. tenulfolla (Pursh) Blake

1020 Asclepiodora zanthodaciyon L. B. Smith

1083 Polyfala alba Nutt. var, tenulfolla (Pursh) Blake

1343 St1pa nimcronata HBR. 
Mueller Mexican Plants Collected 1934.

Determined Carl Epling 1937.

528 Hedeoma nanum (Torr.) Greene subsp. Hexicanum Stewart

481 " Drummond1 Benth.

444 " quercetorum EpI.

385 "

$276 \quad "$

n

$n$ 
SCROPHULARIACEAE COLLECTID IN NUEVO LEON, by

C. H. MUELLER

Buchnera floridana Gand. -- 2052

Castilleja lanata Gray, -- 485, 1109, 2307 ,

$"$ potosina Penn, riz- 1234,2249 ,

" tenuiflora Benth. -- 2095, 2200, 2416,

Pagesia procumbens intermedia Penn., -- 553,

Penstemon cocionophorus Pennel1, - $1245^{\checkmark}, 2266$

" tenuifolius Benth. -- 957, 1188, 2360

Seymeria $\quad-2401$

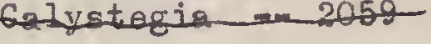

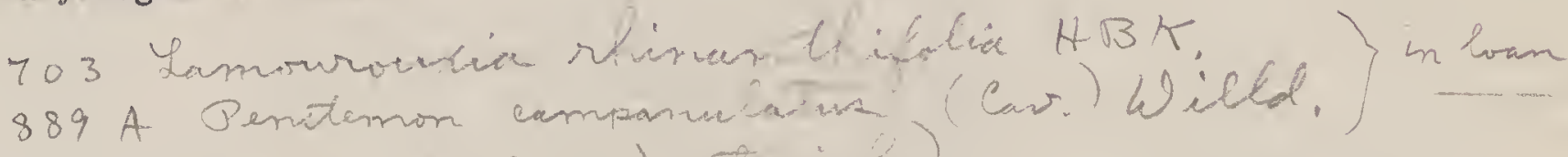

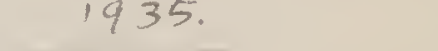

16 mois

out of 30 sent in 1935.

1940 


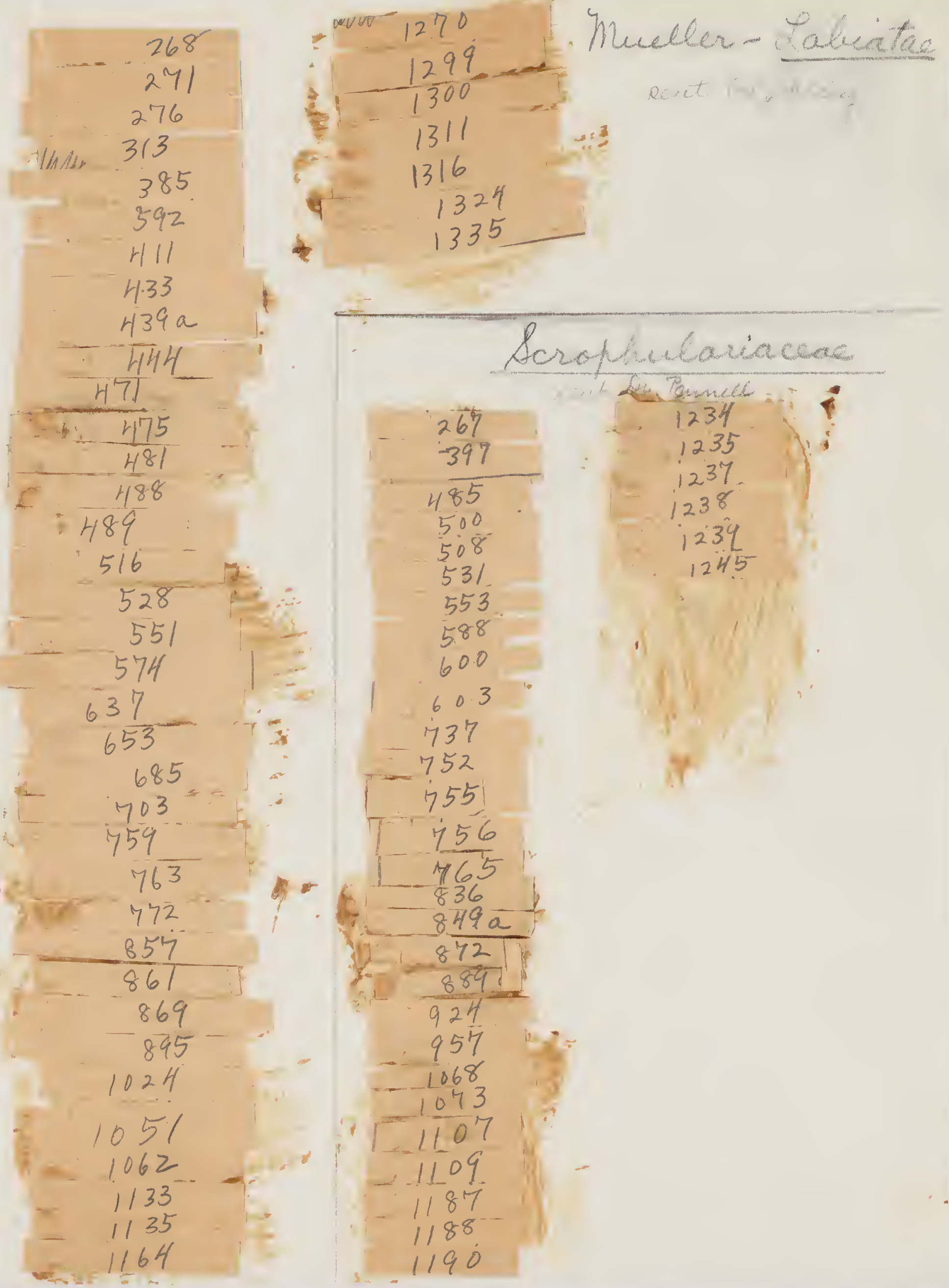

